\title{
The effect of noise, a constant background, and bit depth on the phase retrieval of pure phase objects
}

\author{
VARIS KARITANS $^{1,2^{*}}$, ANDREJS TOKMAKOVS ${ }^{1}$, AdEle ANTONUKA ${ }^{2}$ \\ ${ }^{1}$ Institute of Solid State Physics, University of Latvia, \\ Kengaraga street 8, Riga, Latvia, LV-1063 \\ ${ }^{2}$ Department of Optometry and Vision Science, \\ Faculty of Physics and Mathematics, and Optometry, \\ University of Latvia, Jelgavas street 1, Riga, Latvia, LV-1004 \\ ${ }^{*}$ Corresponding author: Varis.Karitans@cfi.lu.lv
}

\begin{abstract}
In the current study, we investigate the effect of uniform white noise, Poisson noise and a constant background on the phase retrieval of pure phase objects. We also study the influence of the aforementioned factors on phase retrieval at different bit depths of intensity measurements. An algorithm called PhaseLift is used for phase retrieval as it requires a small number of modulating masks and can retrieve the phase of an object from sparse intensity measurements of low bit depth. A test object is modulated by eight random masks generated from a single mask and the phase of the object is retrieved from coded diffraction patterns. Different levels of uniform white noise, Poisson noise and constant background are superimposed on the diffraction patterns and the root-mean-square error (RMSE) of the retrieved object is calculated at each level. The results suggest that Poisson noise and a constant background at the same level cause similar RMSE compared to uniform white noise. Lowering the bit depth from 18-bits to 14-bits resulted in the decrease of the RMSE caused by Poisson noise and a constant background. We conclude that the effects of noise and constant background can be reduced by lowering the bit depth.
\end{abstract}

Keywords: phase retrieval, phase problem, diffraction.

\section{Introduction}

In many fields of science, one often encounters the phase problem, i.e., a detector can only record intensity while the information about phase is lost as there are currently no detectors capable of following the extremely rapid oscillations of the electromagnetic field [1]. The information about phase, however, is very important to retrieve the structure of an object. If the phase of a diffracted field were known, one could retrieve the structure of the diffractive object by simply taking the inverse Fourier transform of the diffraction pattern. The phase problem was first recognised in X-ray analysis to determine 
the structure of crystals [2] ]. It is also experienced in astronomy [ $\underline{3}]$, holography [4], biology [ $[\underline{5}]$ and many other fields of science. In order to overcome the phase problem, various phase retrieval algorithms have been proposed.

Today, coherent diffractive imaging (CDI) is a growing field dealing with phase retrieval from the intensity distribution of the diffracted field [6]. CDI is an attractive field of phase retrieval as it can be easily implemented practically and allows to achieve high resolution of the retrieved object $[\underline{7}, \underline{8}]$. The Gerchberg-Saxton (GS) algorithm was the first algorithm successfully used to solve the phase problem based on alternating projections [9]. Later, FIENUP improved the GS algorithm by replacing the real-space constraints with constraints in agreement with the measured Fourier magnitudes [10]. Two types of the Fienup algorithm are the error-reduction (ER) algorithm and the hybrid input-output (HIO) algorithm [토 $\underline{6}]$. An algorithm called two-step phase retrieval (TSPR), an improvement of the GS algorithm, based on a single-intensity measurement was recently proposed [11]. The TSPR algorithm first retrieves the amplitude of an object using the single-intensity phase retrieval (SIPR) algorithm and then retrieves the phase of the object using the two-intensity phase retrieval (TIPR) algorithm. After the GS algorithm, the well-known phase retrieval method called the transport of intensity equation (TIE) was developed [12]. TIE can retrieve the phase of an object from as small as two intensity measurements at planes placed close to each other. Nowadays, iterative phase retrieval algorithms like PhaseLift $[\underline{13}, \underline{14}]$ and PhaseCut [15] based on optimization techniques have become popular. PhaseLift retrieves the phase of an object from very few diffraction patterns and formulates phase retrieval as finding a rank-one matrix that is subsequently factorised yielding the solution to the phase problem. The original solution of PhaseLift is based on convex optimisation that is very time consuming and computationally intense. Later, a computationally more efficient solution based on low -rank Riemannian optimisation methods was suggested by HuAng et al. [16]. PhaseCut may be seen as a modification of PhaseLift seeking to retrieve the phase of an object directly instead of the whole complex object. Other phase retrieval algorithms like GESPAR [17] and SPAR [ ] ] employing the sparsity of an object were also recently proposed. The algorithm GESPAR retrieving a sparse object from intensity measurements is based on updating the initial random support repeatedly thereby avoiding getting stuck at a local minimum point. The algorithm GESPAR is known to be fast and accurate in the presence of noise and is suited for retrieving the phase of large-scale objects. The algorithm SPAR retrieving a complex object from intensity measurements under noisy conditions makes use of the sparsity of the object and achieves a small reconstruction error and superresolution at the SNR as low as $30 \mathrm{~dB}$.

In the current study, we investigate the algorithm PhaseLift as it has several advantages compared to other algorithms. The algorithms ER and HIO studied by LATYCHEVSKAIA [ 6] require bit depth of at least 16-bits for successful phase retrieval. PhaseLift belongs to the field of low-rank matrix completion and therefore is expected to be able to retrieve the phase of an object from sparse intensity measurements [13] which means low bit depth. This is of particular importance as many commercially 
available cameras have bit depth of 16-bits or 14-bits or even lower [18]. Lowering bit depth may also be seen as a way how to reduce the effect of noise and background on intensity measurements and hence phase retrieval as lower intensity levels corrupted by these undesired effects are forced to zero. When compared to the algorithms SPAR and GESPAR employing the sparsity in the object domain, PhaseLift also has advantages. For an object of size 256 by 256 pixels, SPAR required the computational time on the order of 30 minutes while PhaseLift solved it in several tens of seconds. Regarding the number of masks, in the study [ $[$ ] investigating the algorithm SPAR, twelve masks were used, however, PhaseLift requires a smaller number of masks for successful phase retrieval. CANDÈs et al. observed that at least six or seven masks may be required depending on the type of the masks [14]. HUANG et al. also suggested that six masks may be sufficient for perfect phase retrieval [16]. The algorithm GESPAR, while fast and accurate, retrieves the object in time directly proportional to the third power of the number of non-zero elements [17] while the computational time required by PhaseLift does not depend on the sparsity of an object. It should also be taken into account that many phase retrieval algorithms retrieving the phase of an object from coded diffraction patterns use several random masks all of which are different from each other $[\underline{5}, \underline{8}, \underline{19}]$. Previously, we have shown that all the random modulating masks can be generated from a single random mask by rotating it in different positions [20]. This approach simplifies the design of optical systems practically implementing the algorithm PhaseLift.

Noise with various probability distribution functions (Poisson noise, white noise etc.) is present in imaging systems $[\underline{21}, \underline{22}]$ and affects the accuracy of phase retrieval in CDI as shown by LATYCHEVSKAIA [ 6 ]. A constant background is also present in optical systems, and despite that its role in CDI is well known $[\underline{6}, \underline{23}]$, it has been studied minimally. The purpose of the current study is to evaluate the performance of PhaseLift in the presence of uniform white noise, Poisson noise and a constant background. We also evaluate how the effect of noise and a constant background on the accuracy of phase retrieval depends on the bit depth of intensity measurements.

\section{Methods}

\subsection{Phase retrieval}

The summary of algorithm PhaseLift based on the study by HuANG et al. [16] is further provided. Let us denote a two-dimensional pure phase object to be retrieved by $x_{0} \in \mathbb{C}^{n_{1} \times n_{2}}$ and its vectorized form by $\mathrm{x}_{0} \in \mathbb{C}^{n}$ where $n=n_{1} \cdot n_{2}$. The discrete Fourier transform (DFT) of $x_{0}$ is given by

$$
\mathcal{F}\left(\mathrm{x}_{0}\right)=\mathrm{T} \cdot \mathrm{x}_{0}
$$

where the matrix $\mathrm{T}$ is the Kronecker product of the DFT matrix W, i.e.

$$
\mathrm{T}=\mathrm{W} \otimes \mathrm{W}
$$


Let us also suppose we have $l$ complex random modulating masks $d_{i} \in \mathbb{C}^{n_{1} \times n_{2}}$ where $i=1,2, \ldots, l$. Let us denote the vectorized form of the $d_{i}$ by $\mathrm{d}_{i} \in \mathbb{C}^{n}$ and point-wise multiplication by $\bullet$. The DFT of the modulated object $\mathrm{d}_{i} \bullet \mathrm{x}_{0}$ is given by

$$
\mathcal{F}\left(\mathrm{d}_{i} \bullet \mathrm{x}_{0}\right)=\mathrm{Z}_{i} \cdot \mathrm{x}_{0}
$$

where the matrix $Z_{i}$ is given by an equation

$$
\mathrm{Z}_{i}=\mathrm{T} \cdot \operatorname{Diag}\left(\mathrm{d}_{i}\right)
$$

where $\operatorname{Diag}\left(\mathrm{d}_{i}\right)$ is an $n$-by- $n$ matrix with the modulating waveform $\mathrm{d}_{i}$ on its main diagonal all other elements being zeros.

We can define a matrix $\mathrm{Z}$ for all $l$ masks

$$
\mathrm{Z}=\left(\begin{array}{c}
Z_{1} \\
Z_{2} \\
\vdots \\
Z_{l}
\end{array}\right)
$$

Putting altogether, we can write an expression for the modulus b of the DFT of the modulated object:

$$
\mathrm{b}=\left|\mathrm{Z} \cdot \mathrm{x}_{0}\right|
$$

As only intensity, i.e. the squared modulus of the DFT or Fraunhofer diffraction pattern, is detected, we have quadratic measurements of a form

$$
\mathrm{b}^{2}=\operatorname{diag}\left(\mathrm{Z} \cdot \mathrm{x}_{0} \cdot \mathrm{x}_{0}^{*} \cdot \mathrm{Z}^{*}\right)
$$

where $b^{2}$. denotes the vector containing squared elements of the vector $b, \operatorname{diag}$ (matrix) denotes the vectorial form of the while the asterisk * denotes the conjugate transpose.

If we denote $\mathrm{x}_{0} \cdot \mathrm{x}_{0}^{*}$ by $\mathrm{X}_{0}$, the phase retrieval by PhaseLift can be formulated as finding a rank-one matrix $[\underline{13}-\underline{16}]$ :

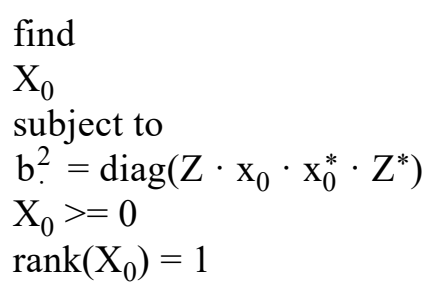

Here, $\mathrm{X}_{0}$ is the rank-one solution and factorizing it in a form $\mathrm{x}_{0} \cdot \mathrm{x}_{0}^{*}$ yields the solution to the phase retrieval problem. The rank minimization problem, however, is NP hard and in convex programming can be relaxed to trace minimization: 


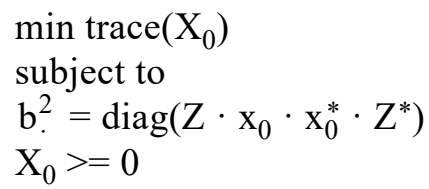

HuANG et al. suggested this using an alternate cost function due to the complexity associated with convex programming [16]. It is known that if the rank of $\mathrm{X}_{0}$ is $p$, then there exists $\mathrm{Y}_{p} \in \mathbb{C}^{n \times p}$ satisfying $\mathrm{Y}_{p} \cdot \mathrm{Y}_{p}^{*}=\mathrm{X}_{0}$. Then the following cost function can be used:

$$
f_{r}\left(\left[\mathrm{Y}_{r}\right]\right)=\frac{\left\|\mathrm{b}^{2}-\operatorname{diag}\left(\mathrm{Z} \cdot \mathrm{Y}_{r} \cdot \mathrm{Y}_{r}^{*} \cdot \mathrm{Z}^{*}\right)\right\|_{2}^{2}}{\left\|\mathrm{~b}^{2}\right\|_{2}^{2}}
$$

where the rank $r$ of the minimizer $\mathrm{Y}_{r} \cdot \mathrm{Y}_{r}^{*}$ is less than or equal to $p$. There is a variety of low-rank Riemannian optimization methods which can be used to optimize the cost function by reducing the rank of the minimizer dynamically [16]. In the current study, the limited memory version of BFGS (LRBFGS) was used to optimize the cost function. The algorithm was stopped when the norm of the gradient of the cost function fell below $10^{-5}$. The algorithm PhaseLift has been implemented into a MATLAB code freely available for download on Internet at the website http://www.math.fsu.edu/ $\sim$ whuang2/papers/SPLRROMCSC.htm. The code was executed on a computer with a CPU Intel ${ }^{\circledR}$ Core $^{\mathrm{TM}} \mathrm{i} 3-2370 \mathrm{M}(2.40 \mathrm{GHz})$ and running the operating system Microsoft ${ }^{\circledR}$ Windows ${ }^{\circledR} 7$.

\subsection{A test object and data processing}

A randomly generated image was created in Corel Photo-Paint X5 and used as a test object. A plane wave of unit amplitude was assumed to illuminate the object. The profile of the surface was expressed in micrometres corresponding to the size of biological cells and fibrils, i.e., in the range of micrometres. The surface profile was then converted to its complex form at the wavelength of a He-Ne laser $(\lambda=0.6328 \mu \mathrm{m})$ corresponding to $2 \pi$ radians. The object was modulated by eight random binary masks modulating only the amplitude of the object. The probability of $0 \mathrm{~s}$ and $1 \mathrm{~s}$ was 0.5 . The size of the object and the mask was 256 by 256 pixels. Only a single mask was used to modulate the object, actually, as four of the masks were rotated versions of the original mask while the other four masks were rotated versions of the flipped version of the original mask. The far-field diffraction patterns were calculated as the squared modulus of the DFT of the modulated object, and the intensity was expressed in relative units.

Uniform white noise with a flat probability distribution function, Poisson noise and a constant background were superimposed on the diffraction patterns in an additive manner and their value was also specified in relative units as part of the maximum value 
of the diffraction pattern. Uniform white noise ranged from zero to the maximum value based on the study by LATYCHEVSKAIA [6]. As noted by LATYCHEVSKAIA, the quality of phase retrieval is significantly compromised when the level of a constant background was on the order of $1 \times 10^{-4}$ of the maximum value of the diffraction pattern. In the current study, the maximum value of uniform white noise was also varied in this range starting from $5 \times 10^{-6}$ to $1 \times 10^{-4}$ (in steps of $5 \times 10^{-6}$ ) of the maximum value of the diffraction pattern. A constant background investigated in the current study was a constant value added to all pixels within the same range. Poisson noise added to the diffraction patterns followed the distribution given by an equation:

$$
p(\mathrm{x} ; \lambda)=\exp (-\lambda) \frac{\lambda^{x}}{x !}
$$

where $p(\mathrm{x} ; \lambda)$ is the probability that the Poisson noise having the parameter $\lambda$ takes the value $x$. The parameter $\lambda$ equal to the mean and variance of the Poisson distribution was varied within the same range as the amplitude of white noise and the level of a constant background.

The influence of noise and a constant background was analysed at three different bit depths: 14-, 16-, and 18-bits. Bit depths 14-bits and 16-bits are among the most common bit depths of raw images of scientific grade cameras [18], however, a bit depth 18 -bits is also found in very advanced cameras. We expect that lowering the bit depth reduces the effect of noise and a constant background by zeroing the lower intensity levels corrupted by noise and a constant background.

The retrieved object was converted back at the wavelength $\lambda=0.6328 \mu \mathrm{m}$ and compared to the test object. Under all conditions, the similarity between the test object and the retrieved object was assessed by calculating the root-mean-square error (RMSE) of the residual phase map or the difference between the test object and the retrieved object. The RMSE of the retrieved object was calculated according to an equation

$$
\operatorname{RMSE}=\sqrt{\frac{1}{n} \sum_{i=1}^{n}\left(\overline{\mathrm{x}}-\mathrm{x}_{i}\right)^{2}}
$$

where $\bar{x}$ is the mean value of the residual phase map while $x_{i}$ is the value of the residual phase map at a given point. $n$ is equal to the total number of sampling points, i.e., 65536 . According to the Eq. (10), the RMSE was measured in the same units as the object, i.e., micrometres $(\mu \mathrm{m})$.

The signal-to-noise ratio (SNR) of intensity measurements was expressed in decibels $(\mathrm{dB})$ using the equation found in the study by HuANG et al. [16]:

$$
\mathrm{SNR}=10 \log _{10}\left(\frac{\left\|\mathrm{b}^{2} \cdot\right\|_{2}^{2}}{\left\|\mathrm{~b}^{2} \cdot \hat{\mathrm{b}}^{2}\right\|_{2}^{2}}\right)
$$

where $b^{2}$ are noise-free intensity measurements while $\hat{b}^{2}$ are noisy intensity measurements. 


\section{Results}

Figure 1 shows the test object and the objects retrieved from noise-free intensity measurements at different bit depths. Despite that noise and background are present in the retrieved objects, visually all the features can be identified. The scale on the right side is shown in micrometres. The RMSE errors of the retrieved objects at bit depths 18,16 , and 14 bits are $0.014,0.022$, and $0.022 \mu \mathrm{m}$, respectively. The computational time was about two minutes while the number of iterations was approx. 1500.

The results suggest that lower bit depth is sufficient for successful phase retrieval with PhaseLift compared to the algorithms ER and HIO. The low bit depth sufficient
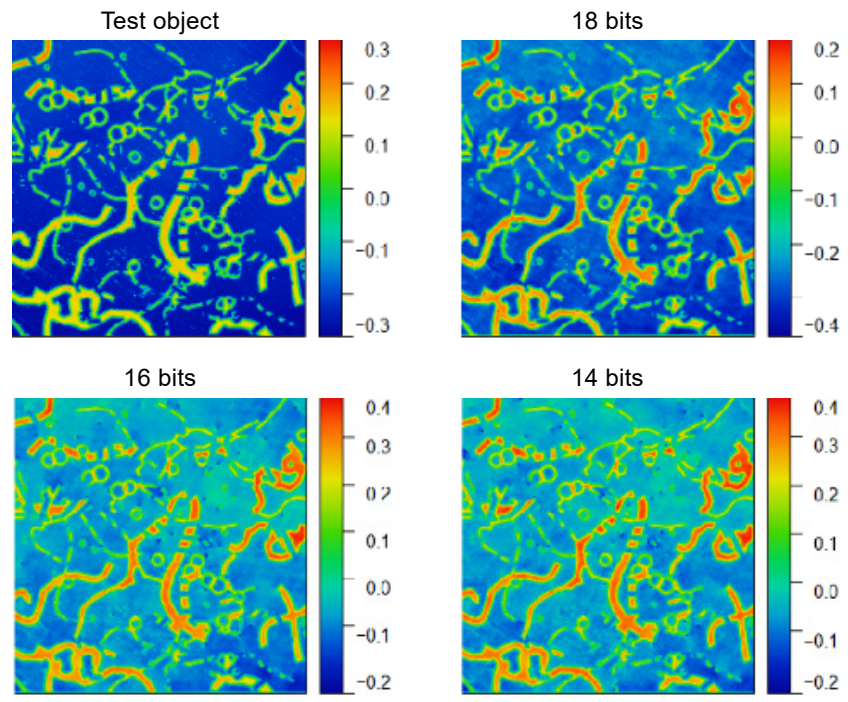

Fig. 1. The test object and the objects retrieved at different bit depths.
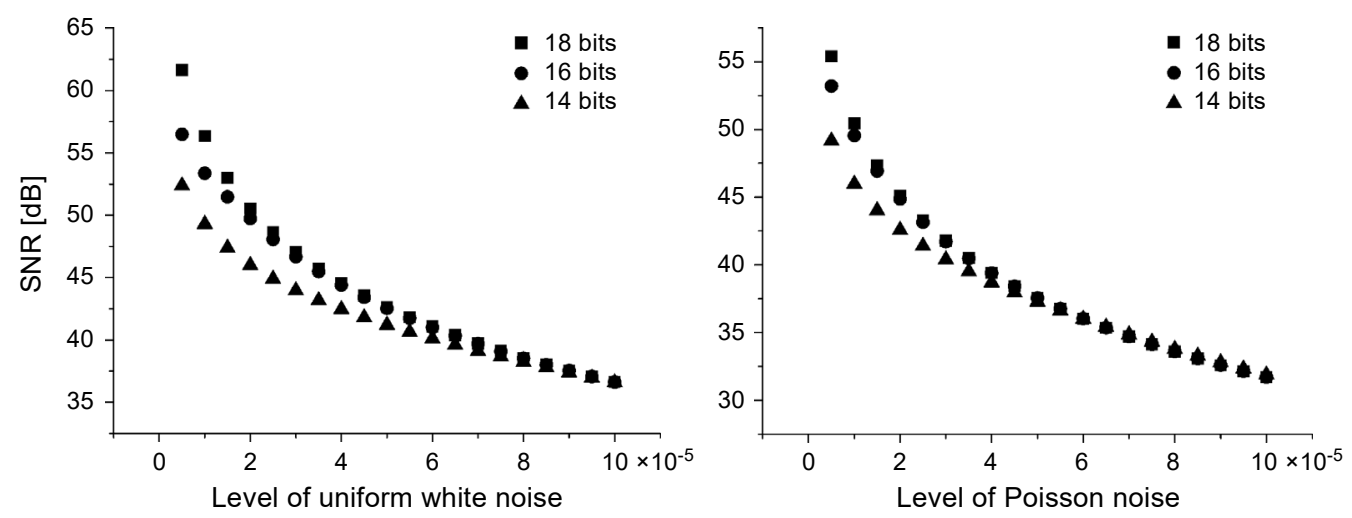

Fig. 2. The dependence of the SNR on the level of uniform white noise and Poisson noise at different bit depths. 
for reasonable phase retrieval demonstrates the ability of PhaseLift to retrieve an object from sparse data as PhaseLift belongs to the field known as low-rank matrix completion.

Figure 2 shows the SNR of intensity measurements both for uniform white noise and Poisson noise as a function of the level of noise expressed as a part of the maximum value of the diffraction pattern. The SNR decreased asymptotically with the increase

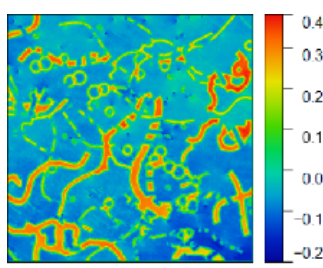

$5 \times 10^{-6}(61.6 \mathrm{~dB})$

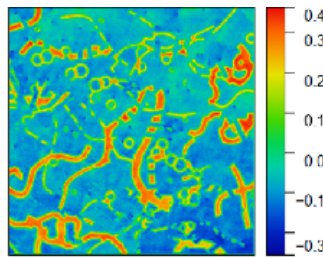

$2.5 \times 10^{-5}(48.6 \mathrm{~dB})$

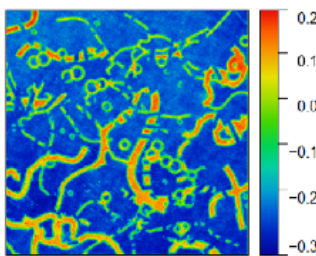

$4.5 \times 10^{-5}(43.6 \mathrm{~dB})$

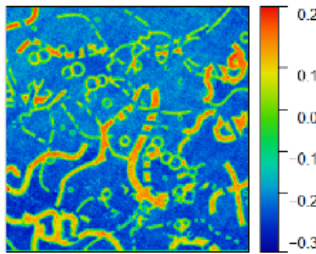

$6.5 \times 10^{-5}(40.4 \mathrm{~dB})$

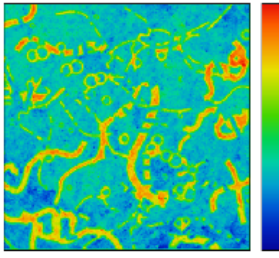

$8.5 \times 10^{-5}(38.0 \mathrm{~dB})$

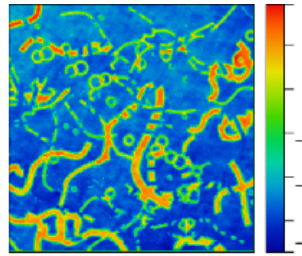

$1 \times 10^{-5}(56.3 \mathrm{~dB})$

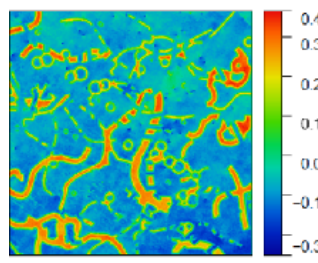

$3 \times 10^{-5}(47.1 \mathrm{~dB})$

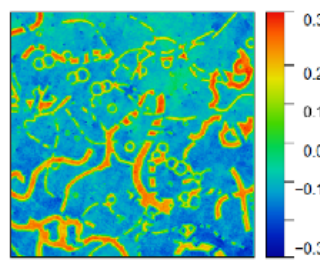

$5 \times 10^{-5}(42.6 \mathrm{~dB})$

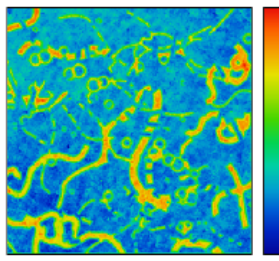

$7 \times 10^{-5}(39.7 \mathrm{~dB})$

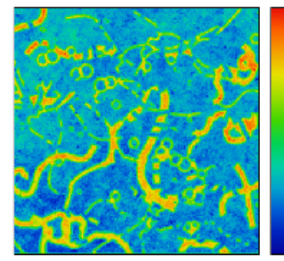

$9 \times 10^{-5}(37.5 \mathrm{~dB})$

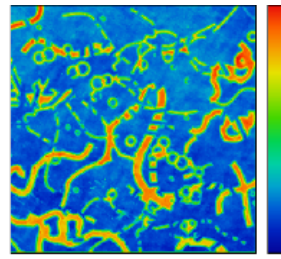

$1.5 \times 10^{-5}(53.0 \mathrm{~dB})$

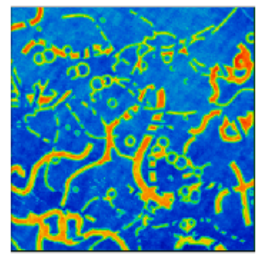

$3.5 \times 10^{-5}(45.7 \mathrm{~dB})$

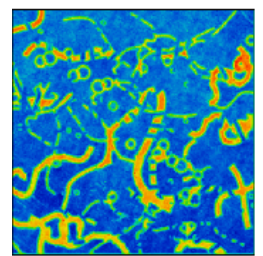

$5.5 \times 10^{-5}(41.8 \mathrm{~dB})$

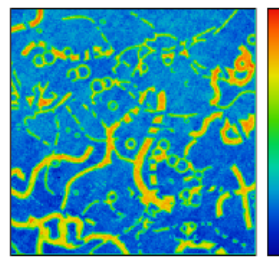

$7.5 \times 10^{-5}(39.1 \mathrm{~dB})$

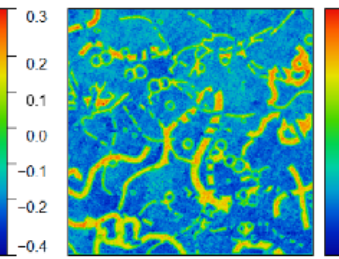

$9.5 \times 10^{-5}(37.1 \mathrm{~dB})$

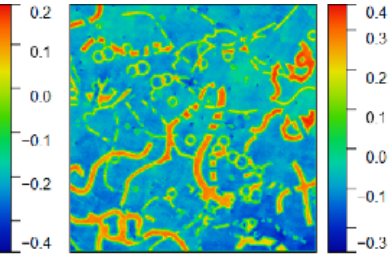

$2 \times 10^{-5}(50.5 \mathrm{~dB})$

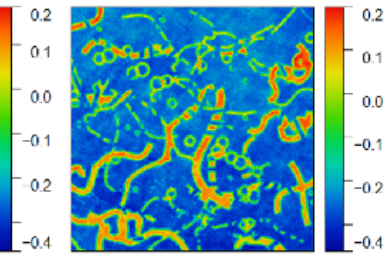

$4 \times 10^{-5}(44.6 \mathrm{~dB})$

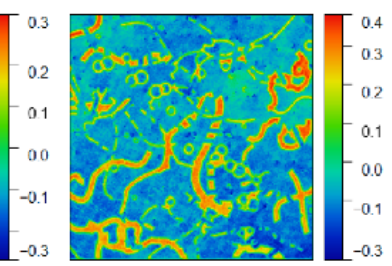

$6 \times 10^{-5}(41.1 \mathrm{~dB})$

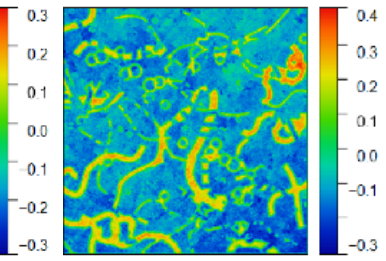

$8 \times 10^{-5}(38.6 \mathrm{~dB})$

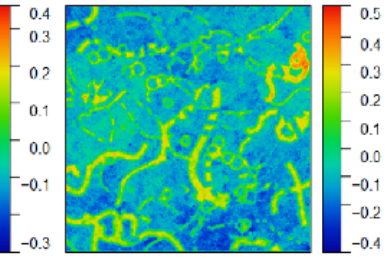

$1 \times 10^{-4}(36.6 \mathrm{~dB})$

Fig. 3. The influence of uniform white noise on phase retrieval. The number below each panel shows the level of uniform white noise as a part of the maximum value of the diffraction pattern and the corresponding SNR of intensity measurements at that level (in brackets). 
in the level of noise, however, the SNR was generally higher for uniform white noise than Poisson noise. As one can notice, at low levels of noise the SNR is considerably decreased as the bit depth is decreased while it becomes almost independent of the bit depth at higher levels of noise.

Figures 3-5 show the retrieved objects at various levels of noise and a constant background. The retrieved objects are shown for the bit depth 18-bits only while the

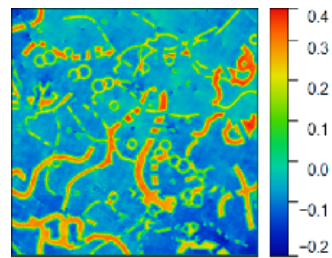

$5 \times 10^{-6}$

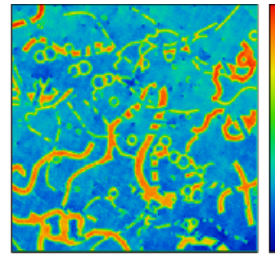

$2.5 \times 10^{-5}$

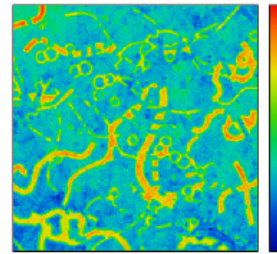

$4.5 \times 10^{-5}$

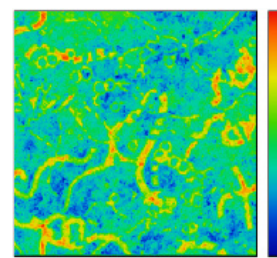

$6.5 \times 10^{-5}$

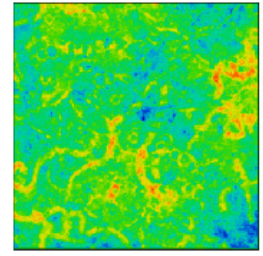

$8.5 \times 10^{-5}$

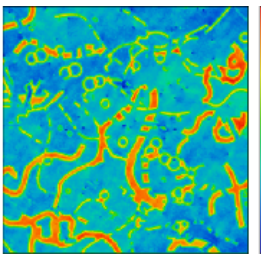

$1 \times 10^{-5}$

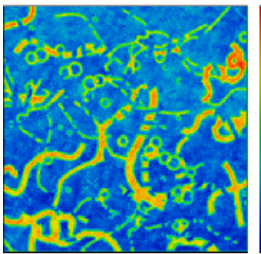

$3 \times 10^{-5}$

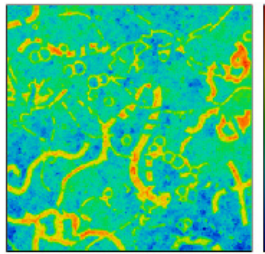

$5 \times 10^{-5}$

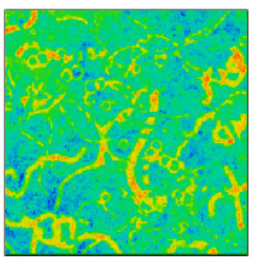

$7 \times 10^{-5}$

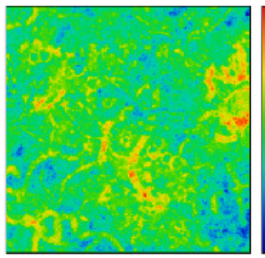

$9 \times 10^{-5}$

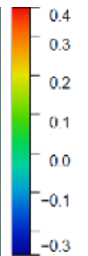

3

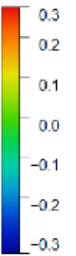

$-0.3$

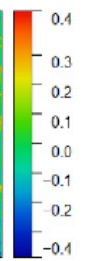

$-0.4$

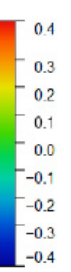

$-0.4$

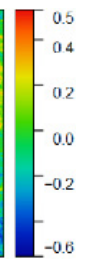

\begin{abstract}
fluence of a constant bac
\end{abstract}
Fig. 4. The influence of a constant background on phase retrieval. The number below each panel shows the level of a constant background as a part of the maximum value of the diffraction pattern.

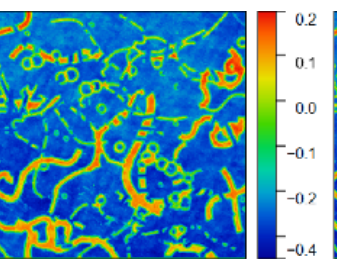

$1.5 \times 10^{-5}$

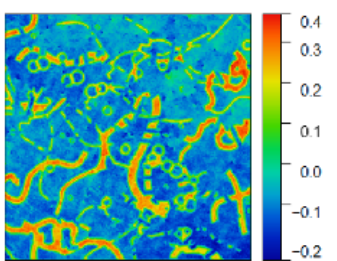

$3.5 \times 10^{-5}$

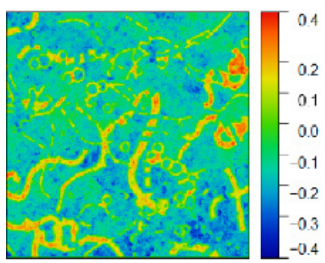

$5.5 \times 10^{-5}$

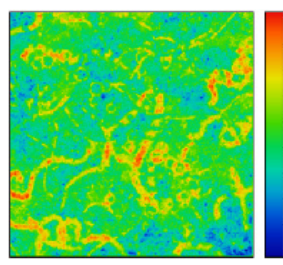

$7.5 \times 10^{-5}$

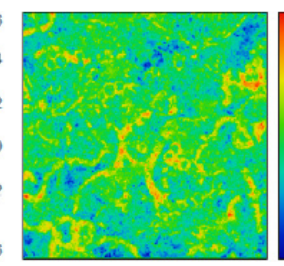

$9.5 \times 10^{-5}$

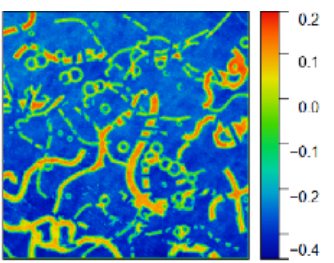

$2 \times 10^{-5}$

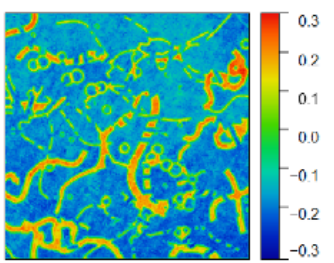

$4 \times 10^{-5}$

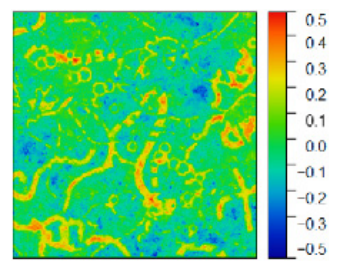

$6 \times 10^{-5}$

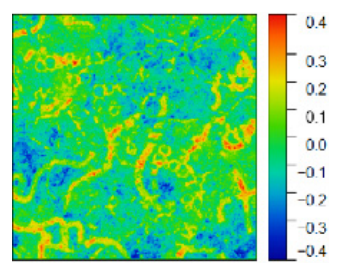

$8 \times 10^{-5}$

$1 \times 10^{-4}$

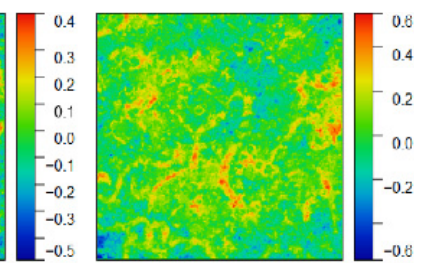




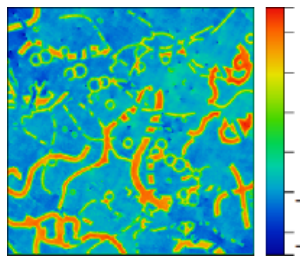

$5 \times 10^{-6}(55.4 \mathrm{~dB})$

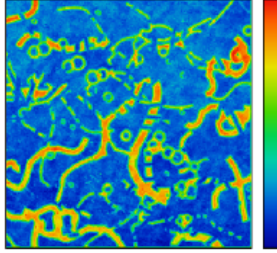

$2.5 \times 10^{-5}(43.3 \mathrm{~dB})$

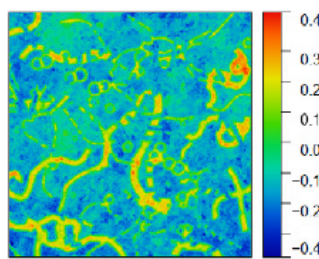

$4.5 \times 10^{-5}(38.4 \mathrm{~dB})$

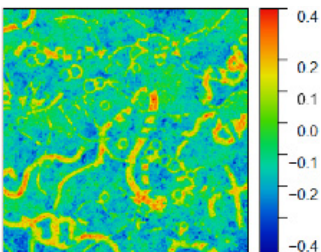

$6.5 \times 10^{-5}(35.4 \mathrm{~dB})$

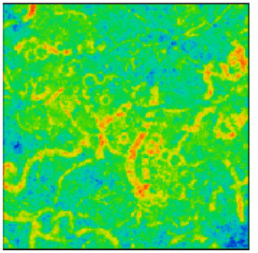

$8.5 \times 10^{-5}(33.1 \mathrm{~dB})$

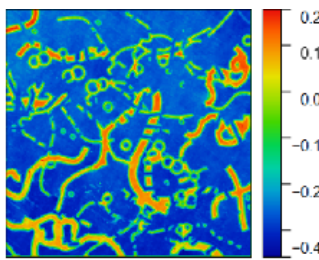

$1 \times 10^{-5}(50.5 \mathrm{~dB})$

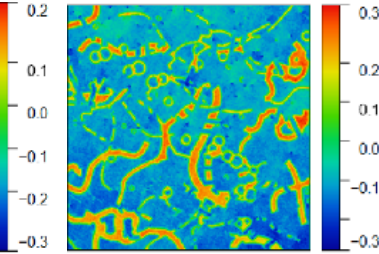

$3 \times 10^{-5}(41.8 \mathrm{~dB})$

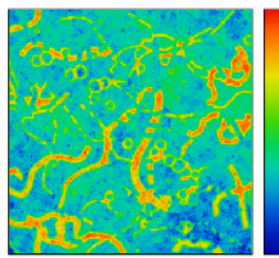

$5 \times 10^{-5}(37.6 \mathrm{~dB})$

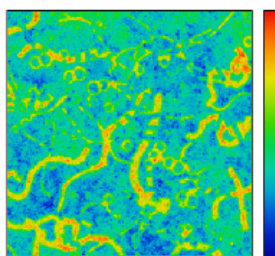

$7 \times 10^{-5}(34.7 \mathrm{~dB})$

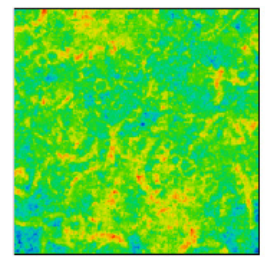

$9 \times 10^{-5}(32.6 \mathrm{~dB})$

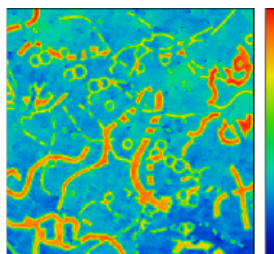

$1.5 \times 10^{-5}(47.4 \mathrm{~dB})$

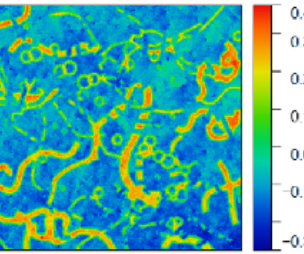

$3.5 \times 10^{-5}(40.5 \mathrm{~dB})$

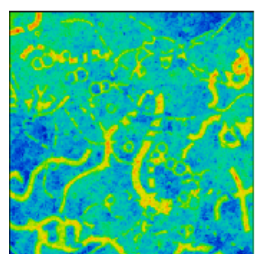

$5.5 \times 10^{-5}(36.8 \mathrm{~dB})$

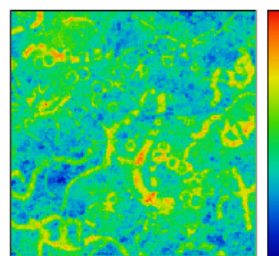

$7.5 \times 10^{-5}(34.1 \mathrm{~dB})$

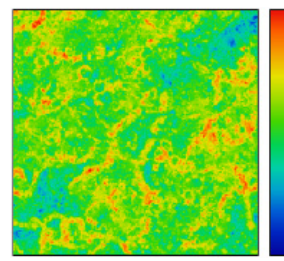

$9.5 \times 10^{-5}(32.1 \mathrm{~dB})$

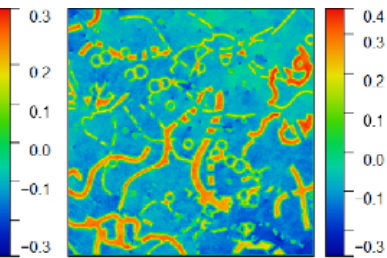

$2 \times 10^{-5}(45.1 \mathrm{~dB})$

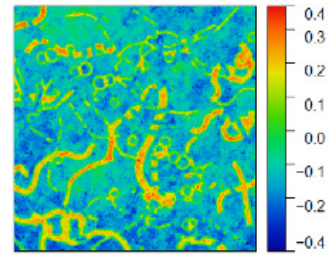

$4 \times 10^{-5}(39.4 \mathrm{~dB})$

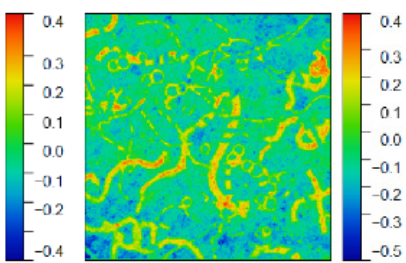

$6 \times 10^{-5}(36.0 \mathrm{~dB})$

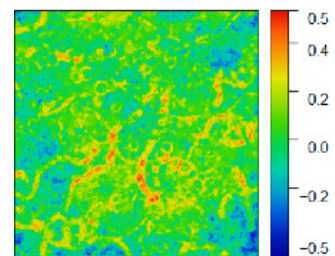

$8 \times 10^{-5}(33.6 \mathrm{~dB})$

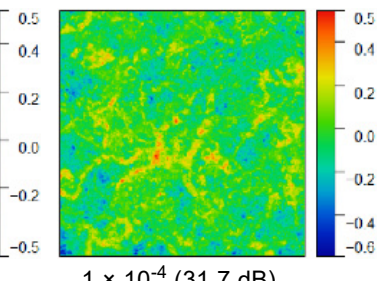

Fig. 5. The influence of Poisson noise on phase retrieval. The number below each panel shows the level of Poisson noise as a part of the maximum value of the diffraction pattern and the corresponding SNR of intensity measurements at that level (in brackets).

analysis of the SNR (Fig. 2) and RMSE (Fig. 6) is shown for all bit depths. Figure 3 shows the effect of uniform white noise on phase retrieval. The two numbers below each panel show the level of uniform white noise as a part of the maximum value of the diffraction pattern and the corresponding SNR of intensity measurements in $\mathrm{dB}$ (in brackets). The features of the retrieved objects can be easily recognised even at high 
levels of noise. Figure 4 shows the effect of a constant background on phase retrieval. The number below each panel again shows the level of a constant background as a part of the maximum value. It can be noted that starting from the level of a constant background $\left(9 \times 10^{-5}\right)$ the features of the retrieved object become almost indistinguishable. By comparing Figs. 3 and 4 it can be concluded that at one and the same level of uniform white noise and a constant background, a larger detrimental effect on phase retrieval is caused by the background. Figure 5 shows the effect of Poisson noise on phase retrieval. The two numbers below each panel show the value of the parameter $\lambda$ as a part of the maximum value of the diffraction pattern and the corresponding SNR of intensity measurements in $\mathrm{dB}$ (in brackets). At all levels of Poisson noise and a constant background, the effects on phase retrieval are similar and much larger than those caused by uniform white noise.

Figure 6 shows the RMSE in the case of uniform white noise, Poisson noise and a constant background at all bit depths. It is obvious that at all bit depths, uniform white noise causes the smallest RMSE compared to Poisson noise and a constant background closely following each other. While the RMSE is similar at the bit depths 18-bits and 16-bits, lowering the bit depth down to 14-bits considerably reduces the RMSE at high levels of Poisson noise and a constant background.
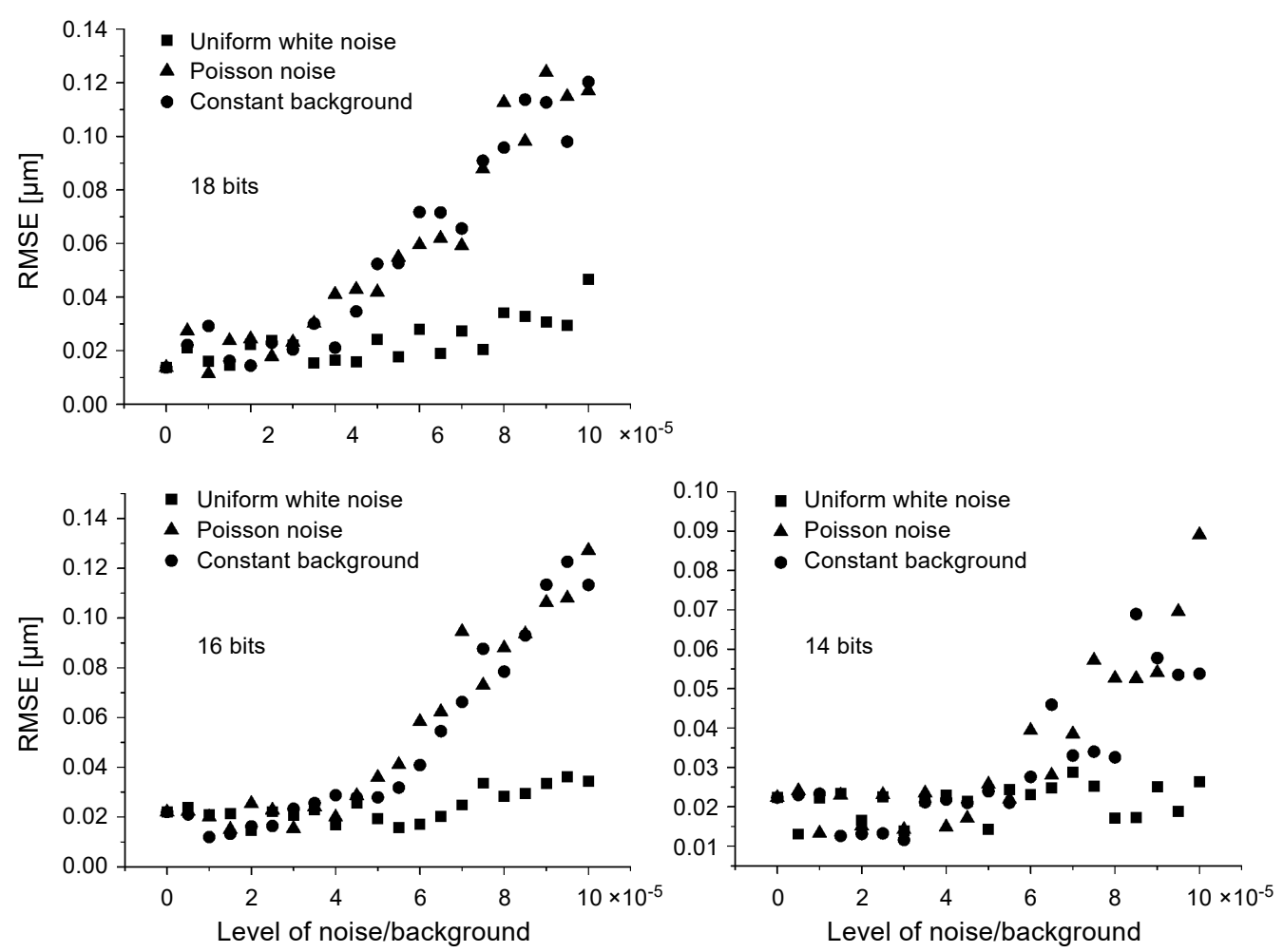

Fig. 6. The RMSE as a function of the level of uniform white noise, a constant background and Poisson noise at different bit depth. 


\section{Conclusions}

The simulations demonstrate that PhaseLift can retrieve the phase of an object by modulating its amplitude with different versions of one and the same mask. In the case of noise-free intensity measurements, the noise and background present in the retrieved objects may be due to the small number of modulating masks, however, the optical design based on a single mask remains attractive from the viewpoint of a simple optical design. The results confirm that different types of noise and a constant background compromise the quality of phase retrieval to a different extent. At one and the same level of Poisson noise and a constant background and bit depths 18-bits and 16-bits, the RMSEs are almost equal and larger than those caused by uniform white noise. Uniform white noise, however, is seldom present in optical systems and is usually found in a combination with Poisson noise [13, 14], especially at low light levels, and a constant background.

Despite that the SNR was decreased as bit depth was decreased, especially at low levels of noise, the RMSE also decreased due to the zeroing of the intensity levels corrupted by noise and a constant background. It is known that the phase retrieval of multidimensional objects benefits from oversampling in the Fourier domain $[\underline{1}, \underline{13}]$. The adverse effects of noise and a constant background may be reduced by oversampling in the Fourier domain, however, this increases the computational load as oversampling in Fourier domain requires zero padding in the real domain thereby reducing the advantages of PhaseLift compared to other phase retrieval algorithms.

Acknowledgements - The authors are supported by the postdoctoral project (1.1.1.2/16/I/001; 1.1.1.2/ VIAA/1/16/199, State Education Development Agency, Republic of Latvia).

\section{References}

[1] Shechtman Y., Eldar Y.C., Cohen O., Chapman H.N., Miao J., Segev M., Phase retrieval with application to optical imaging: a contemporary overview, IEEE Signal Processing Magazine 32(3), 2015, pp. 87-109, DOI: 10.1109/MSP.2014.2352673.

[2] Harrison R.W., Phase problem in crystallography, Journal of the Optical Society of America A 10(5), 1993, pp. 1046-1055, DOI: 10.1364/JOSAA.10.001046.

[3] DainTy J.C. [Ed.], Laser Speckle and Related Phenomena, Springer, Berlin, Heidelberg, 1975, pp. 255-280.

[4] Barmherzig D.A., Sun J., Li P.-N., LANe T.J., CAndès E.J., Holographic phase retrieval and reference design, Inverse Problems 35(9), 2019, article 094001, DOI: 10.1088/1361-6420/ab23d1.

[5] Shevkunov I., Katkovnik V., Petrov N.V., Egiazarian K., Super-resolution microscopy for biological specimens: lensless phase retrieval in noisy conditions, Biomedical Optics Express 9(11), 2018, pp. 5511-5523, DOI: 10.1364/BOE.9.005511.

[6] LATYCHEVSKAIA T., Iterative phase retrieval in coherent diffractive imaging: practical issues, Applied Optics 57(25), 2018, pp. 7187-7197, DOI: 10.1364/AO.57.007187.

[7] Szameit A., Shechtman Y., Osherovich E., Bullkich E., Sidorenko P., Dana H., Steiner S., Kley E.B., Gazit S., Cohen-Hyams T., Shoham S., Zibulevsky M., Yavneh I., Eldar Y.C., Cohen O., Segev M., Sparsity-based single-shot subwavelength coherent diffractive imaging, Nature Materials 11(5), 2012, pp. 455-459, DOI: 10.1038/nmat3289. 
[8] Katkovnik V., Egiazarian K., Sparse superresolution phase retrieval from phase-coded noisy intensity patterns, Optical Engineering 56(9), 2017, article 094103, DOI: $10.1117 / 1.0 E .56 .9 .094103$.

[9] Gerchberg R.W., Saxton W.O., A practical algorithm for the determination of phase from image and diffraction plane pictures, Optik 35(2), 1972, pp. 237-246.

[10] FiENuP J.R., Phase retrieval algorithms: a comparison, Applied Optics 21(15), 1982, pp. 2758-2769, DOI: $10.1364 / \mathrm{AO} .21 .002758$.

[11] Zhang C., Wang M., Chen Q., WAng D., Wei S., Two-step phase retrieval algorithm using single -intensity measurement, International Journal of Optics, Vol. 2018, 2018, article 8643819, DOI: $10.1155 / 2018 / 8643819$.

[12] Teague M.R., Deterministic phase retrieval: a Green's function solution, Journal of the Optical Society of America 73(11), 1983, pp. 1434-1441, DOI: 10.1364/JOSA.73.001434.

[13] Candès E.J., Eldar Y.C., Strohmer T., Voroninski V., Phase retrieval via matrix completion, SIAM Journal on Imaging Sciences 6(1), 2013, pp. 199-225, DOI: 10.1137/110848074.

[14] Candès E.J., Li X., Soltanolkotabi M., Phase retrieval from coded diffraction patterns, Applied and Computational Harmonic Analysis 39(2), 2015, pp. 277-299, DOI: 10.1016/j.acha.2014.09.004.

[15] Waldspurger I., D’Aspremont A., Mallat S., Phase recovery, MaxCut and complex semidefinite programming, Mathematical Programming 149(1-2), 2015, pp. 47-81, DOI: $10.1007 /$ s10107-013-0738-9.

[16] Huang W., Gallivan K.A., Zhang X., Solving PhaseLift by low-rank Riemannian optimization methods for complex semidefinite constraints, SIAM Journal on Scientific Computing 39(5), 2017, pp. B840-B859, DOI: 10.1137/16M1072838.

[17] Shechtman Y., Beck A., Eldar Y.C., GESPAR: efficient phase retrieval of sparse signals, IEEE Transactions on Signal Processing 62(4), 2014, pp. 928-938, DOI: 10.1109/TSP.2013.2297687.

[18] Cheremkhin P.A., Krasnov V.V., Kurbatova E.A., Rodin V.G., Starikov S.N., Estimation of number of resolvable signal levels of photo- and videocameras, Journal of Physics: Conference Series 536(1), 2014, article 012023, DOI: 10.1088/1742-6596/536/1/012023.

[19] Seaberg M.H., D’Aspremont A., Turner J.J., Coherent diffractive imaging using randomly coded masks, Applied Physics Letters 107(23), 2015, article 231103, DOI: 10.1063/1.4937122.

[20] Karitans V., Nitiss E., Tokmakovs A., Ozolinsh M., Logina S., Optical phase retrieval using four rotated versions of a single binary amplitude modulating mask, Journal of Astronomical Telescopes, Instruments, and Systems 5(3), 2019, article 039004, DOI: 10.1117/1.JATIS.5.3.039004.

[21] Brouk I., Nemirovsky A., Nemirovsky Y., Analysis of noise in CMOS image sensor, Proceedings of the 2008 IEEE International Conference on Microwaves, Communications, Antennas and Electronic Systems, May 13-14, 2008, Tel-Aviv, Israel, IEEE, 4562800, DOI: 10.1109/COMCAS.2008.4562800.

[22] Boyat A.K., Joshi B.K., A review paper: noise models in digital image processing, Signal \& Image Processing: An International Journal 6(2), 2015, pp. 63-75.

[23] Migukin A., Agour M., Katкovnik V., Phase retrieval in 4 foptical system: background compensation and sparse regularization of object with binary amplitude, Applied Optics 52(1), 2013, pp. A269 -A280, DOI: 10.1364/AO.52.00A269. 